\title{
Non-redundant functions of two proline dehydrogenase isoforms in Arabidopsis
}

\author{
Dietmar Funck*, Sonja Eckard and Gudrun Müller
}

\begin{abstract}
Background: Proline (Pro) accumulation is a widespread response of prokaryotic and eukaryotic cells subjected to osmotic stress or dehydration. When the cells are released from stress, Pro is degraded to glutamate by Prodehydrogenase (ProDH) and Pyrroline-5-carboxylate dehydrogenase (P5CDH), which are both mitochondrial enzymes in eukaryotes. While P5CDH is a single copy gene in Arabidopsis, two ProDH genes have been identified in the genome. Until now, only ProDH1 (At3g30775) had been functionally characterised.

Results: We demonstrate vasculature specific expression of the Arabidopsis ProDH2 gene (At5g38710) as well as enzymatic activity and mitochondrial localisation of the encoded protein. Expression levels of ProDH2 are generally low, but increased in senescent leaves and in the abscission zone of floral organs. While sucrose represses ProDH2 expression, Pro and $\mathrm{NaCl}$ were identified as inducers. Endogenous ProDH2 expression was not able to overcome Pro sensitivity of ProDH1 mutants, but overexpression of a GFP-tagged form of ProDH2 enabled the utilisation of Pro as single nitrogen source for growth. Amongst two intronic insertion mutants, one was identified as a null allele, whereas the other still produced native ProDH2 transcripts.

Conclusions: Arabidopsis possesses two functional ProDHs, which have non-redundant, although partially overlapping physiological functions. The two ProDH isoforms differ with respect to spatial, developmental and environmental regulation of expression. While ProDH1 appears to be the dominant isoform under most conditions and in most tissues, ProDH2 was specifically upregulated during salt stress, when ProDH1 was repressed. The characterisation of $\mathrm{ProDH} 2$ as a functional gene requires a careful re-analysis of mutants with a deletion of ProDH1, which were so far considered to be devoid of ProDH activity. We hypothesise that $\mathrm{ProDH} 2$ plays an important role in Pro homeostasis in the vasculature, especially under stress conditions that promote Pro accumulation.
\end{abstract}

\section{Background}

Arabidopsis, like most plant species, accumulates free proline (Pro) to high concentration in response to salt and drought stress. The physiological function of Pro is subject to controversial debate, and Arabidopsis plants with a reduced capacity to accumulate Pro showed only a moderate decrease or no change in stress tolerance [1-3]. Pro is suggested to act as a compatible osmolyte, a stabilising agent for macromolecules, a radical scavenger or as a nitrogen and energy store for the recovery phase $[4,5]$. Other hypotheses attribute the beneficial function to the process of Pro metabolism rather than the mere accumu-

* Correspondence: dietmar.funck@uni-konstanz.de

1 Department of Plant Physiology and Biochemistry, Biology Section, University of Konstanz, Universitätsstrasse 10, 78464 Konstanz, Germany

Full list of author information is available at the end of the article lation of Pro. Along this line, energy transfer between cellular compartments, regeneration of electron acceptors or signalling functions have been proposed $[6,7]$.

The biochemistry of Pro biosynthesis and degradation has been intensively studied and most of the participating proteins and the genes encoding for these enzymes were characterised with respect to temporal and spatial expression patterns and enzymatic properties (Fig. 1; [5]). The first step of Pro synthesis is catalysed by Pyrroline-5carboxylate synthetase (P5CS), which uses glutamate, NADPH and ATP to generate glutamate- $\gamma$-semialdehyde (GSA). GSA is in spontaneous equilibrium with the cyclic pyrroline-5-carboxylate (P5C), which is converted to Pro by $\mathrm{P} 5 \mathrm{C}$ reductase $(\mathrm{P} 5 \mathrm{CR})$, again consuming $\mathrm{NADPH}$. Arabidopsis contains two isoforms of P5CS that are dif- 


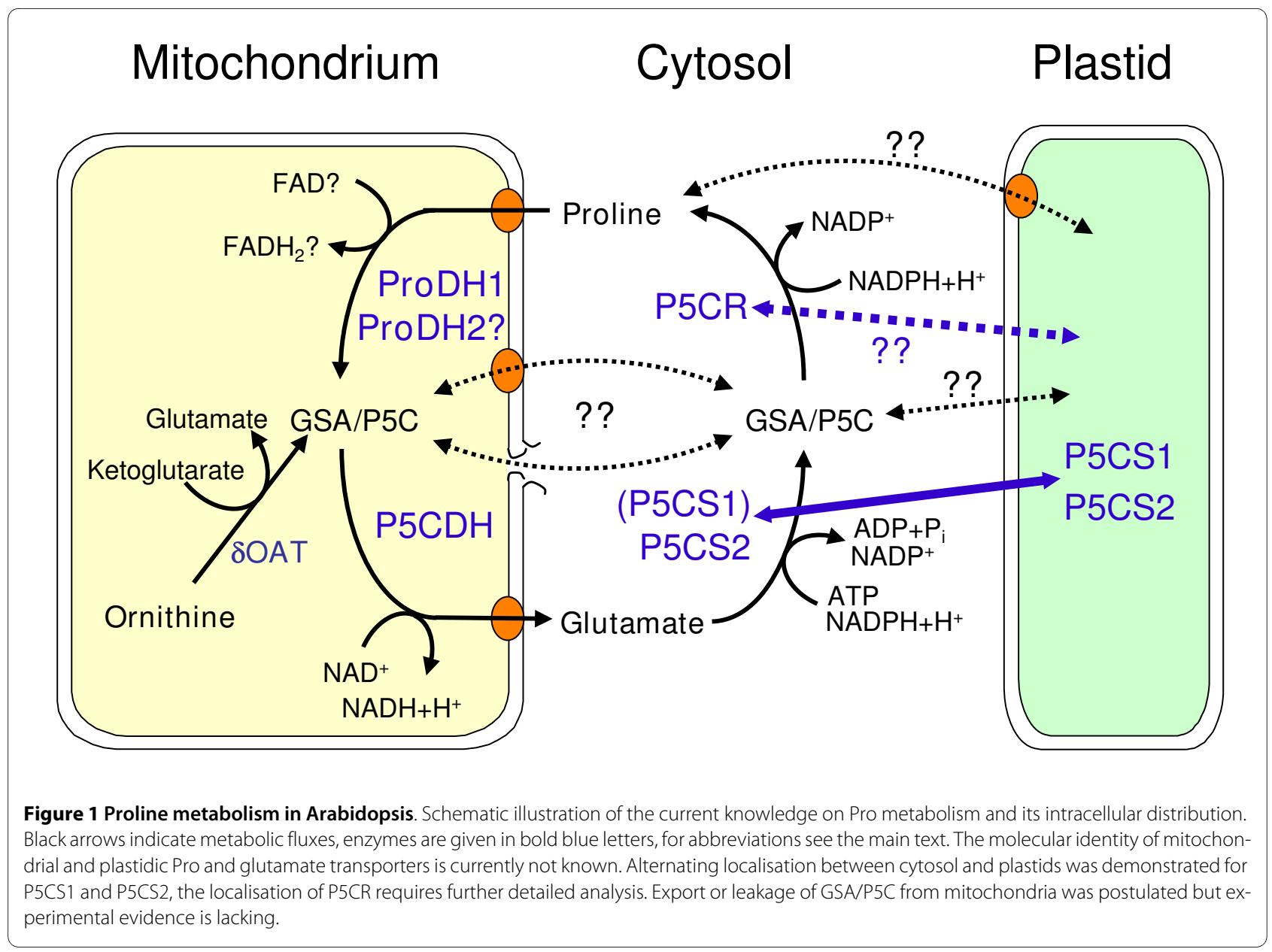

ferentially regulated. P5CS2 (At3g55610) expression was consistent with a housekeeping function, and the P5CS2 protein was found to be localised in the cytosol under normal conditions while being partially re-located to plastids during stress $[3,8,9]$. Pro accumulation under stress conditions is primarily contingent upon induction of P5CS1 (At2g39800) expression. Also P5CS1 was observed in the cytosol, partly as presumably inactive aggregates, and re-localised to plastids during stress [3]. P5CR seems to be predominantly localised in the cytosol, while some activity was also associated with plastid preparations $[10,11]$. However, direct investigations of the localisation and enzymatic properties of Arabidopsis P5CR (At5g14800) are still pending. A second pathway for Pro synthesis from ornithine had also been postulated, but characterisation of Ornithine- $\delta$-aminotransferase indicated that ornithine is degraded to glutamate inside the mitochondria prior to its conversion to Pro by the standard pathway $[12,13]$. Loss or gain of function mutants provided valuable evidence for the physiological role of Pro accumulation, although the interpretation of the mutant phenotypes was always hampered by the primary function of Pro in protein biosynthesis [4].
For degradation, Pro is imported into the mitochondria, where it is converted back to glutamate by the tightly coupled activities of Pro dehydrogenase (ProDH) and $\mathrm{P} 5 \mathrm{C}$ dehydrogenase (P5CDH $[14,15])$. In Arabidopsis, one ProDH (ProDH1; At3g30775) and one P5CDH (At5g62530) have been characterised at the molecular level. ProDH1 and P5CDH expression is repressed by osmotic stress and upregulated by Pro, with the changes being more pronounced for ProDH1 [4]. Also in flowers, strong expression of ProDH1 was observed in the stigma and in pollen, while $P 5 C D H$ is only upregulated in pollen $[16,17]$. Mutants with defects in Pro degradation grew without obvious phenotypic differences under greenhouse conditions, only a slight decrease in seed quality was noted for $p 5 c d h$ mutants [16,18-20]. Common to the p5cdh mutants and the ProDH1 mutant pdh1-1 were an enhanced and prolonged accumulation of Pro during stress and an unexpected hypersensitivity to external Pro supply in the absence of stress. In wildtype plants, external application of harmful amounts of Pro caused the loss of mitochondrial and plastid integrity [21]. Hare et al. (2002) hypothesised that excess Pro degradation would cause misdirection of electrons to $\mathrm{O}_{2}$ in mitochondria 
and plastids due to electron overflow or acceptor limitation, respectively. Recent results confirmed the Prodependent production of reactive oxygen species (ROS) in mitochondria, an effect that was more pronounced in ProDH overexpressing or $p 5 c d h$ mutant plants [22].

The only obvious gap in the molecular characterisation of Pro metabolism in Arabidopsis remains a second isoform of ProDH (ProDH2, At5g38710), which has been identified as an expressed gene with high homology to ProDH1 [1,23]. The predicted pre-proteins of ProDH1 and ProDH2 share 75\% identical amino acids (aa). Hanson et al. (2008) demonstrated a direct induction of $\mathrm{ProDH} 2$ by the sucrose-repressed transcription factor bZIP11 [24]. However, until now the enzymatic functionality of ProDH2 had not been addressed. Here, we demonstrate that ProDH2 expressed in yeast can mediate Pro degradation equal to ProDH1. Overexpression of a ProDH2-GFP construct in Arabidopsis was able to rescue the Pro-hypersensitive phenotype of the pdh1-1 knockout mutant. We detected ProDH2-GFP in the mitochondria and analysis of GUS expression under control of the ProDH2 promoter demonstrated specific expression in the vascular tissue and in the abscission zone of petals, sepals and stamina. In striking contrast to ProDH1, ProDH2 expression was induced by salt stress and was absent in reproductive tissues.

\section{Results}

\section{At5g38710 encodes a protein with ProDH activity}

Reports on successful determination of ProDH activity from plant mitochondrial membranes are very rare. A variety of papers ascribe a soluble activity of Pro dependent $\mathrm{NAD}^{+}$reduction at $\mathrm{pH} 10.3$ to $\mathrm{ProDH}$, but this is more likely to be the reverse reaction of P5CR (Giuseppe Forlani, unpublished results). Therefore we choose to test the enzymatic function of the putative ProDH2 (At5g38710) by heterologous expression in yeast. To obtain a suitable test system, we generated a mutant yeast strain in which the Put1 gene, encoding the single ProDH, was replaced by a kanamycin and geneticin resistance cassette by homologous recombination. The resulting geneticin resistant strain $\Delta p u t 1$ was unable to catabolise Pro as a source of nitrogen for growth (Fig. 2). Transformation of the $\Delta p u t 1$ strain with a plasmid harbouring the full-length cDNA of At5g38710 did not enable the cells to metabolise Pro. A similar observation for ProDH1 has been described by Kiyosue et al. (1996) and was confirmed by our results [25]. Exchange of the predicted mitochondrial transit peptide (mTP) of the putative ProDH2 (aa 1-29) against the mTP of the flavoprotein subunit of yeast succinate dehydrogenase $(S c S d h 1)$ lead to the expression of a protein that rescued the Pro metabolism defect of the $\Delta$ put 1 strain. Also an exchange of the first 39 amino acid residues of ProDH1
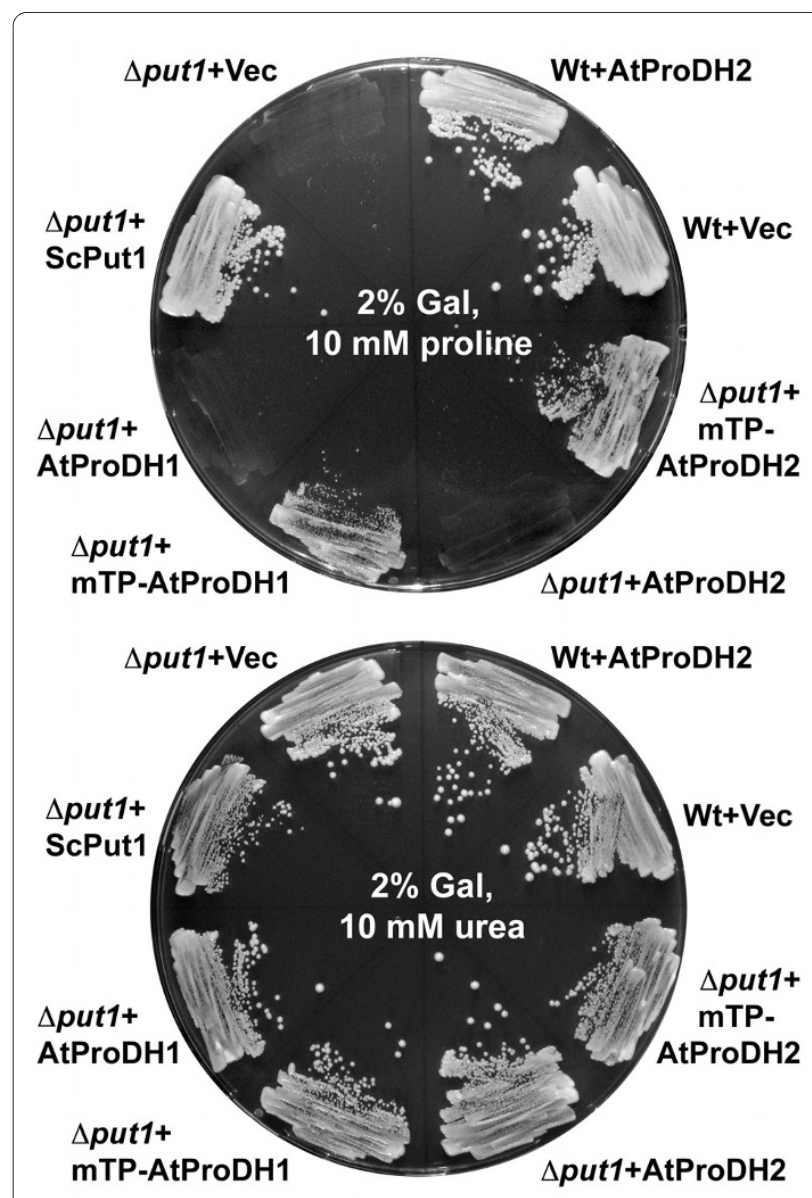

Figure 2 ProDH 2 expression complements a yeast $\Delta p u t 1$ mutant. Yeast wildtype or $\triangle$ put1 mutant with various ProDH expression constructs were grown for $4 \mathrm{~d}$ on minimal medium with $2 \%(\mathrm{w} / \mathrm{v})$ galactose (Gal) as inducing carbon source and $10 \mathrm{mM}$ Pro (upper plate) or $10 \mathrm{mM}$ urea (lower plate) as the sole nitrogen source. Expression of ScPut1 confers wildtype growth to the $\triangle p u t 1$ mutant. Native AtProDH pre-proteins do not complement the Pro utilisation deficiency of the $\triangle$ put 1 mutant. Replacement of the predicted Arabidopsis mTPs by the mTP of the yeast $S d h 1$ gene confers functional expression and the capability to metabolise external Pro as N-source (mTP-AtProDH1 and mTP-AtProDH2).

against the $S c S d h 1$-mTP enabled the expression of a functional protein. The $S c S d h 1$-mTP was chosen because it is unlikely to interfere with the functionality test for the Arabidopsis ProDHs. From these results we concluded that the name ProDH2 was justified for At5g38710. Under non-inducing conditions, with glucose instead of galactose as carbon source, none of the ProDH1 or ProDH 2 expression constructs enabled the $\Delta p u t 1$ strain to catabolise Pro (data not shown). Expression of the fulllength ProDH2 cDNA in wildtype yeast did not alter the growth properties and western blot analysis of cells expressing a ProDH2 protein with $6 \times$ His-tag confirmed the presence of the recombinant protein (Fig. 2 and data not shown). These findings indicate that the native 
ProDH2 protein is not harmful to yeast, but does not have sufficient ProDH activity to enable utilisation of Pro as nitrogen source.

\section{ProDH2 is expressed specifically in the vascular tissue}

The presence of two ProDH genes in the Arabidopsis genome, which both encode functional ProDHs, raised the question of the specific function of ProDH2. Expression analysis of ProDH1 indicated ubiquitous expression in all organs, with the highest expression levels detected in pollen grains and in the stigma of the carpel [17]. To compare the expression of ProDH1 and ProDH2, we fused $1500 \mathrm{bp}$ of upstream sequence of the $\mathrm{ProDH} 2$ gene to a $\beta$-glucuronidase (GUS) gene. Bioinformatic analysis of $3000 \mathrm{bp}$ of genomic sequence upstream of the start codon of ProDH2 with the Athena analysis tool did not reveal any potential transcription factor binding sites that were more abundant than the expectations for random distribution would predict $[26,27]$. However, consensus motifs for an abscisic acid responsive element and a drought responsive element were identified at -880 and $1395 \mathrm{bp}$, respectively. Most of the putative binding motifs that were found between -1500 and $-3000 \mathrm{bp}$ were also present in the region between -1 and $-1500 \mathrm{bp}$. Therefore we chose to include only the region up to $-1500 \mathrm{bp}$ in the GUS-construct. Transgenic plants harbouring this construct expressed GUS activity exclusively in the vascular tissue (Fig. 3A-C). Of the 30 analysed primary transformants, all showed a very similar expression pattern, albeit the amount of GUS activity detected by X-Gluc staining varied considerably (Data not shown). Staining was generally weak in the youngest leaves and strongest in the older leaves. For further analysis, plants with intermediate activity in mature leaves were chosen. In inflorescences, the ProDH2-promoter induced GUS expression only in the abscission zone immediately below the pistils or siliques. This high expression persisted through silique maturation, demonstrating a partial spatial separation of ProDH1 and ProDH2 expression in flowers. Northern blot analysis of RNA isolated from various tissues of soil grown Arabidopsis wildtype plants confirmed that the expression of $\mathrm{ProDH} 2$ is stronger in floral organs (Fig. $3 \mathrm{D}$ ). In young (less than $7 \mathrm{~mm}$ long) and mature leaves (more that $3 \mathrm{~cm}$ long), no ProDH2 transcripts were detected, presumably due to the low relative content of vascular tissue, whereas a strong signal in senescent leaves (showing anthocyanin accumulation and visible reduction in chlorophyll content) confirmed age-dependent regulation of $\mathrm{ProDH} 2$ expression. In petioles from mature leaves, no ProDH2 expression was detected by northern blot although this part of the leaf should be enriched in vascular tissue. Also in roots and leaf-derived cell cultures, a strong expression of ProDH2 was detected. In contrast, ProDH1 was expressed also in a root-derived cell culture and was only slightly induced in senescent leaves. In the inflorescence, ProDH1 expression

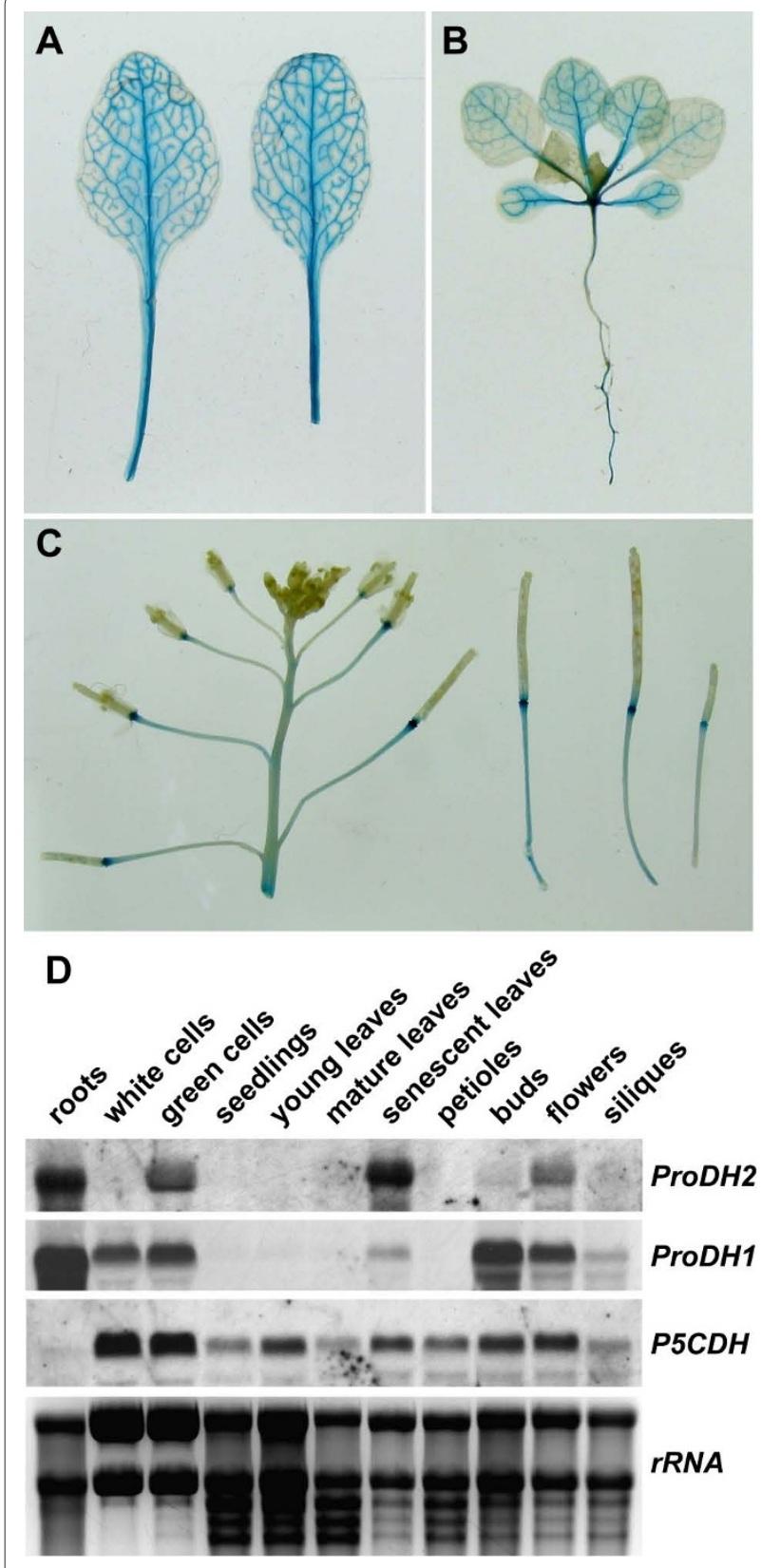

Figure 3 Tissue distribution of $\mathrm{ProDH} 2$ expression. A-C: $\mathrm{PrODH} 2-$ promoter activity visualised in transgenic plants carrying a $\mathrm{ProDH} 2$ promoter-Gus fusion construct. Tissues were stained for $6 \mathrm{~h}$ in $1 \mathrm{mMX}$ Gluc and destained in $80 \%(\mathrm{v} / \mathrm{v}) \mathrm{EtOH}$. A: Two mature leaves of a 6week-old plant. B: Three-week-old seedling showing enhanced staining with progressing leaf age. C: Inflorescence and young siliques showing GUS activity in the abscission zone between the pistils and the floral stalk. D: Northern blot analysis of transcript levels of Pro degradation genes in various tissues and two different cell cultures. The picture of the EtBr stained gel is shown to demonstrate RNA integrity and equal loading.

reached its peak before $\mathrm{ProDH}$ 2. $\mathrm{P} 5 \mathrm{CDH}$ was detected in all organs analysed. However, a surprisingly low transcript level of $P 5 C D H$ was observed in roots, where both ProDH isoforms were strongly expressed. 


\section{ProDH2 is induced by proline and salt but repressed by sugar}

Since the variation of GUS activity between different transgenic lines was high, we used northern blot analysis to investigate the regulation of ProDH2 expression. Hanson et al. (2008) identified ProDH2 as a direct target of the transcription factor bZIP11 [24]. In agreement with these findings, ProDH2 transcript levels were higher when plants were incubated in medium without sucrose compared to treatment with 30 or $100 \mathrm{mM}$ sucrose (Fig. 4). Also incubation for $6 \mathrm{~h}$ in medium containing $20 \mathrm{mM}$ Pro or $200 \mathrm{mM} \mathrm{NaCl}$ in addition to $30 \mathrm{mM}$ sucrose enhanced ProDH2 expression slightly. These concentrations were found to be sub-lethal during the period of the treatment and effective in triggering changes in Pro metabolism. Induction of ProDH2 by salt stress was surprising, since ProDH1 is repressed under these conditions. Induction of P5CS1 expression by $200 \mathrm{mM} \mathrm{NaCl}$ demonstrated that the treatment was efficient in triggering Pro accumulation. In general, the signals obtained for ProDH2 were near the detection limit, while ProDH1 detection yielded much stronger signals. This observation was confirmed by analysis of publicly available microarray data, in which $\mathrm{ProDH} 2$ expression is fre-

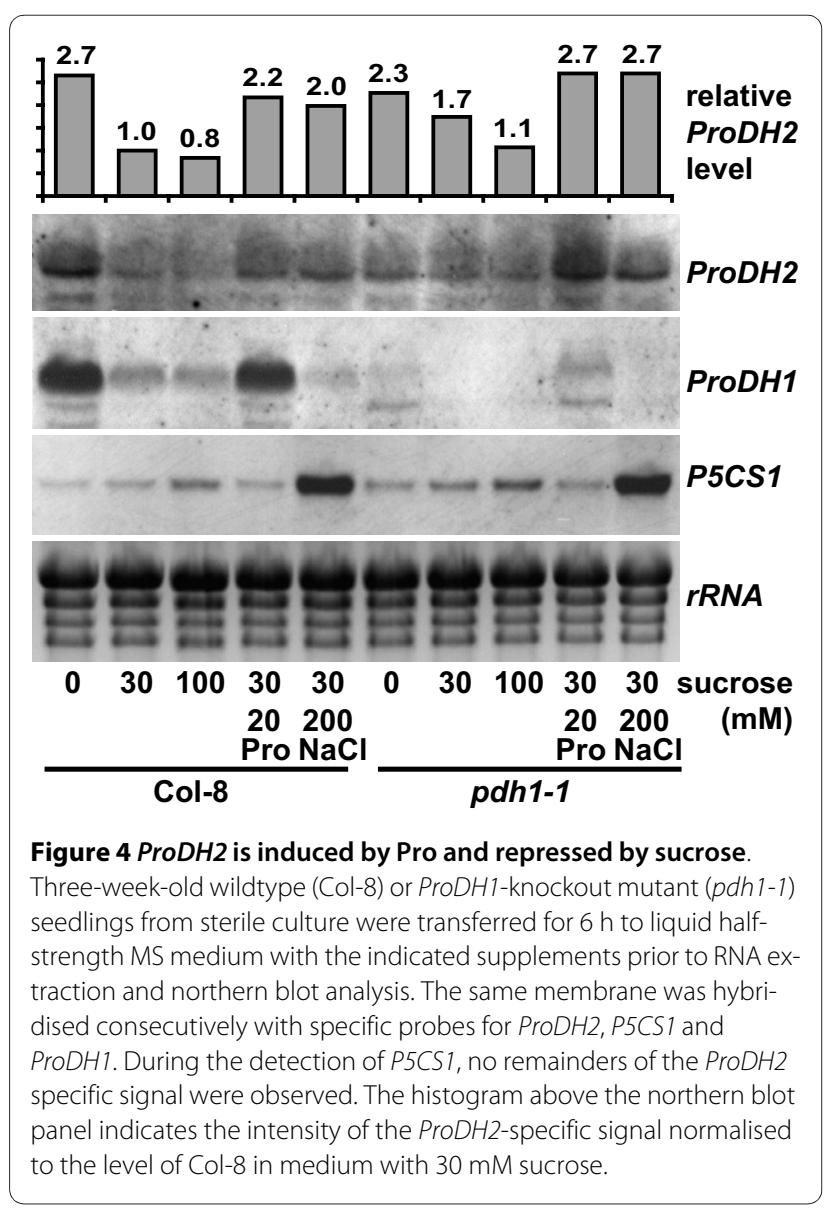

quently not clearly detected (data not shown [28,29]). For example, in the AtGenExpress stress series, the maximal signal intensity for ProDH1 is 7.7-fold higher than for ProDH2. In the same dataset, the ProDH1 expression pattern was more similar to $\mathrm{P} 5 \mathrm{CDH}$ than to ProDH2. Pathogen challenge and drought stress were among the rare cases, in which microarray analyses detected a stronger expression of ProDH2 than of ProDH1.

We also analysed potential compensatory changes of ProDH2 expression in the ProDH1 knockout mutant pdh1-1 [20]. Basal levels and sugar-dependent regulation of ProDH2 were only mildly affected in the pdh1-1 mutant. Induction of $\mathrm{ProDH} 2$ by Pro was slightly enhanced, presumably by the higher intracellular Pro content caused by lack of the ProDH1 isoform, which seems to be predominant in most tissues of wildtype plants. P5CS1 transcript levels were the same in wildtype and pdh1-1 mutant plants, while ProDH1 transcripts were mostly undetectable in the mutant. Under conditions of ProDH1 induction, some aberrant transcripts were also detected in $p d h 1-1$.

\section{A role of ProDH2 in Pro tolerance}

The low and spatially limited expression of ProDH2 posed the question of whether the ProDH2 protein can make a significant contribution to Pro metabolism. Therefore we directly compared the Pro sensitivity of pdh1-1, pdh1-4 (see below) and p5cdh-2 mutants, reasoning that Pro degradation is completely blocked in p5cdh-2 mutants, while $p d h 1$ mutants still contain the active ProDH2 isoform. As described earlier, neither of these mutants was able to use $5 \mathrm{mM}$ Pro as the sole source of nitrogen (Fig. 5A). Seedlings of both mutant genotypes did not expand properly and stayed etiolated, while wildtype seedlings turned green and developed true leaves. Compared to Col-8, the Ler ecotype showed a decreased ability to de-etiolate and grow with Pro as the only nitrogen source. A transposon insertion mutant devoid of ProDH2 (pdh2-1; see below) did not show increased sensitivity compared to the parental Ler ecotype.

When $p d h 1$ and $p 5 c d h$ mutants were assayed for Pro sensitivity in the presence of mineral nitrogen, only p5cdh-2 mutants showed a dose dependent inhibitory effect of Pro on hypocotyl elongation (cell elongation) and fresh weight accumulation (cell growth) that was different from the wildtype (Fig 5B and data not shown). These findings indicate that expression of ProDH2 is not sufficient to compensate fully for the lack of ProDH1, but may still be able to confer limited Pro tolerance in the presence of mineral nitrogen. Contrary to the loss of ProDH1 or P5CDH expression, loss of ProDH2 did not result in Pro hypersensitivity. 


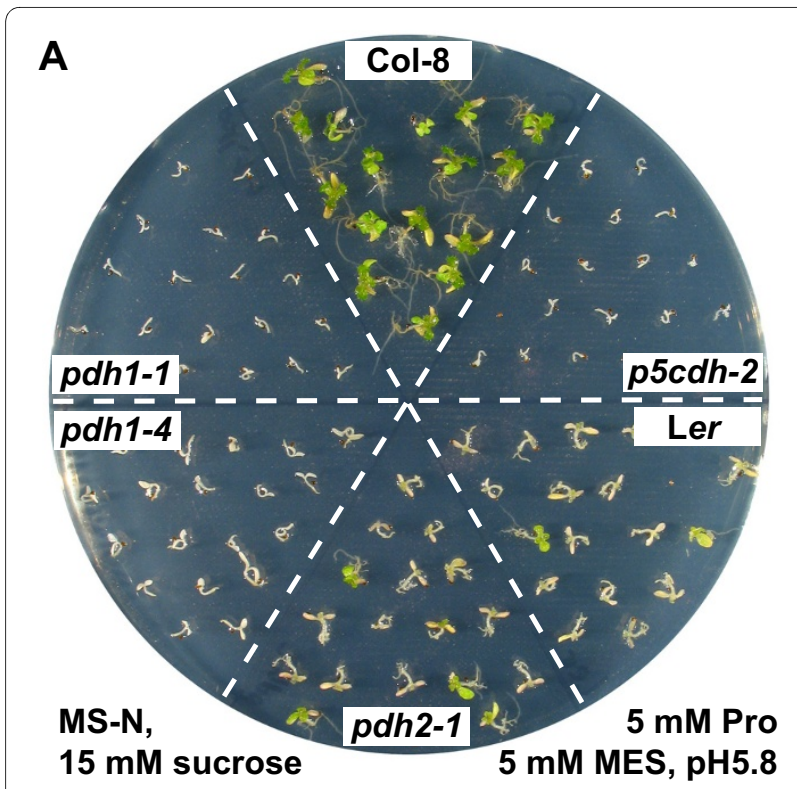

B

Hypocotyl elongation $1 / 2 \mathrm{MS}, 30 \mathrm{mM}$ sucrose

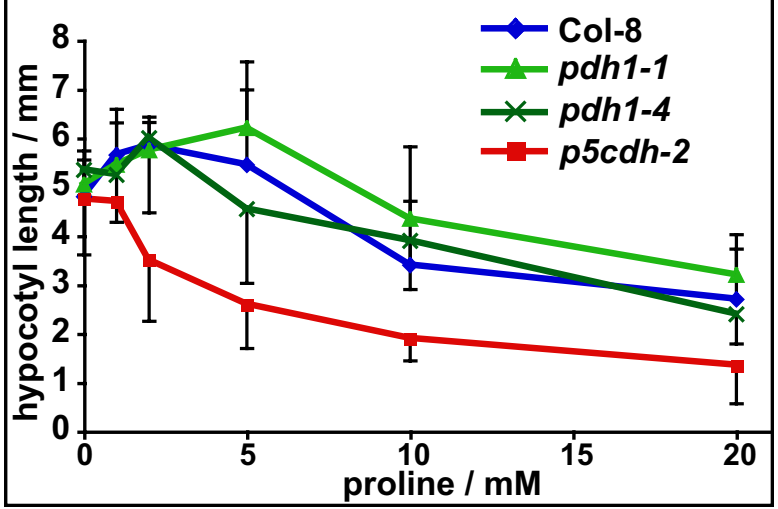

Figure $5 p 5 c d h-2$ mutants are more sensitive to external Pro supply than pdh1 mutants. A: Col-8 and Ler wildtypes, $p d h 1-1, p d h 1-4$, $p d h 2-1$ and $p 5 c d h-2$ seedlings were cultivated for 2 weeks on MS medium with $5 \mathrm{mM}$ Pro as the sole source of nitrogen and $15 \mathrm{mM}$ sucrose as carbon source, buffered to pH 5.8 with 5 mM MES. B: Hypocotyl elongation of Col-8 wildtype, $p d h 1-1, p d h 1-4$ and $p 5 c d h$-2 seedlings cultivated on MS medium supplemented with $30 \mathrm{mM}$ sucrose and varying concentrations of Pro. Error bars indicate $S D$ of $\geq 10$ seedlings per genotype. The experiments were repeated with similar results.

Robust splicing of T-DNAs in the first intron of ProDH1 and ProDH2

To enable a direct comparison of mutants completely devoid of ProDH activity to the $p 5 c d h-2$ mutant, we screened the public T-DNA insertion lines for $p d h 2$ mutants. Several lines (SALK_108179, GABI_918D08, SAIL_90_G05, GABI_187C05) with T-DNA insertions immediately upstream or downstream of the ProDH2 coding sequence had little or no effect on transcript abundance (data not shown). Two lines were identified with a T-DNA or a transposon in the first intron of
ProDH2 (GABI_328G05 and GT1788, respectively). By northern blot and RT-PCR analysis we were unable to detect native transcripts in homozygous GT1788 plants, while both aberrant and a small amount of native transcripts were present in GABI_328G05 (Fig. 6 and data not shown). Therefore we termed these two insertion-lines pdh2-1 and pdh2-2, respectively. Histochemical analysis revealed no detectable GUS activity in pdh2-1 plants, indicating that the GUS gene of gene-trap construct contained in the transposon is not in frame with the first ProDH2 exon after potential splicing events (data not shown). A similar analysis of ProDH1 insertion lines confirmed the absence of native transcripts in pdh1-1, SALK_081276 (pdh1-2) and GABI_308F08 (pdh1-3). In line SALK_119334 (pdh1-4), which carries an inverted tandem repeat of the T-DNA in the first intron of ProDH1, a mixture of aberrant and native transcripts was detected, similar to the $p d h 2-2$ insertion line. When grown under greenhouse conditions, all ProDH1 or ProDH2 insertion lines showed no phenotypic differences to the corresponding wildtypes (data not shown). The production of useful $p d h 1 / p d h 2$ double mutants is impeded by the fact that the insertion line $p d h 2-1$ is in Ler background, in which stress-responsive Pro accumulation and Pro sensitivity differs from Col-8 (Fig. 5A and data not shown). Therefore, double mutants will have to be compared to multiple families of Col-8/Ler hybrids or crossed several times to one of the parental ecotypes.

\section{ProDH2 is localised to mitochondria}

The reason for the inability of ProDH1 mutants to utilise Pro as nitrogen source, despite a functional ProDH 2 copy, could be that ProDH2 is not in the correct subcellular localisation to support Pro degradation. Prediction programs clearly indicated a mitochondrial localisation, which we analysed with the help of transgenic plants expressing a ProDH2-GFP fusion protein. The GFP signals obtained from such plants were much weaker compared to ProDH1-GFP expression driven by the same promoter, but both ProDH2-GFP and ProDH1-GFP were clearly localised in mitochondria (Fig. 7 and Additional File 1). Subcellular localisation and enzymatic functionality of ProDH2 indicated that the low expression level and/ or the tissue specificity were responsible for the inability of pdh1-1 mutants to utilise Pro as nitrogen source.

\section{ProDH2-GFP overexpression rescues the pdh1-1 mutant}

To test the functional equivalence of ProDH2 and ProDH1, also the pdh1-1 mutant was transformed with the constructs for the 35S-driven expression of ProDH1GFP and ProDH2-GFP. Overexpression of ProDH2-GFP or ProDH1-GFP restored the capability of $p d h 1-1$ mutants to use Pro as the sole source of nitrogen (Fig. 8). These findings indicate that tissue specificity and expres- 


\section{A}
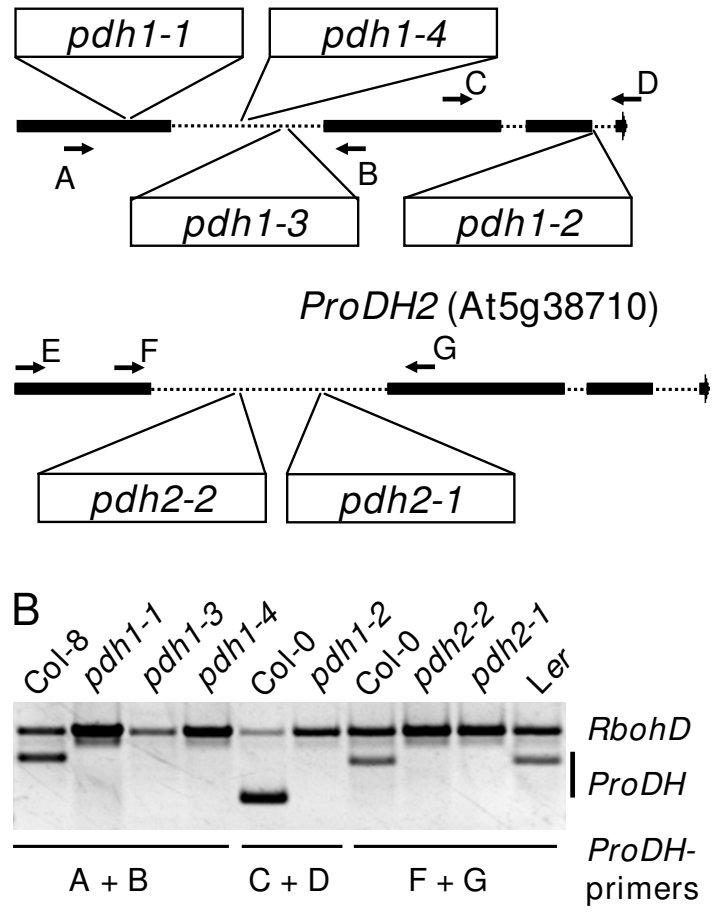

$\mathrm{C}$

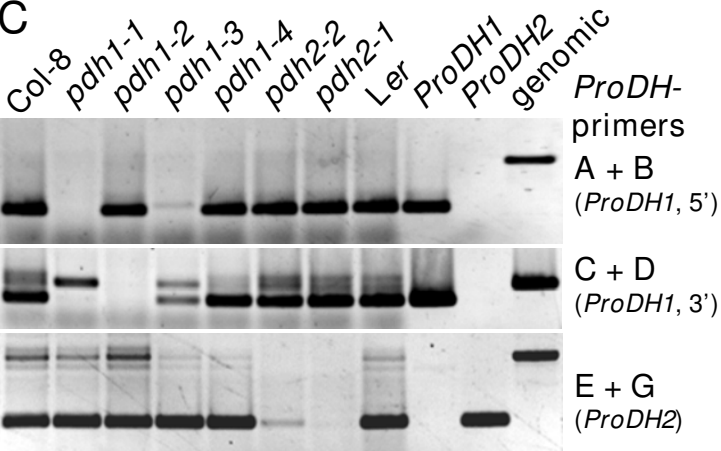

Figure 6 Knockout lines for ProDH1 and ProDH2 and T-DNA excision during splicing. A: Graphic representation of the exon-intron structure of the ProDH1 and ProDH2 genes with the position of T-DNA insertions in the analysed mutant lines (see material and methods section). Arrows with letters indicate the binding sites of primers used for $P C R$ analyses in $B$ and C. B: PCR on genomic DNA with two primer pairs, one for RbohD (At5g47910 as internal control, fragment size 1272 bp) and one ProDH1 or ProDH2 specific primer pair. Absence of ProDH specific PCR products demonstrates that all mutant plants were homozygous for the respective T-DNA insertion. C: RT-PCR analysis of ProDH1 and $\mathrm{ProDH} 2$ expression in the mutant lines demonstrates the presence of native transcripts in $p d h 1-4$ and $p d h 2-2$. PCR reactions with cloned cDNAs and genomic DNA (leftmost three lanes) demonstrate specificity of the PCR products. CDNAs for $C$ and genomic DNA for $B$ were obtained from the same samples, a slight contamination of the RNA samples with genomic DNA caused the additional amplification of intron-containing PCR products in the RT-PCRs.
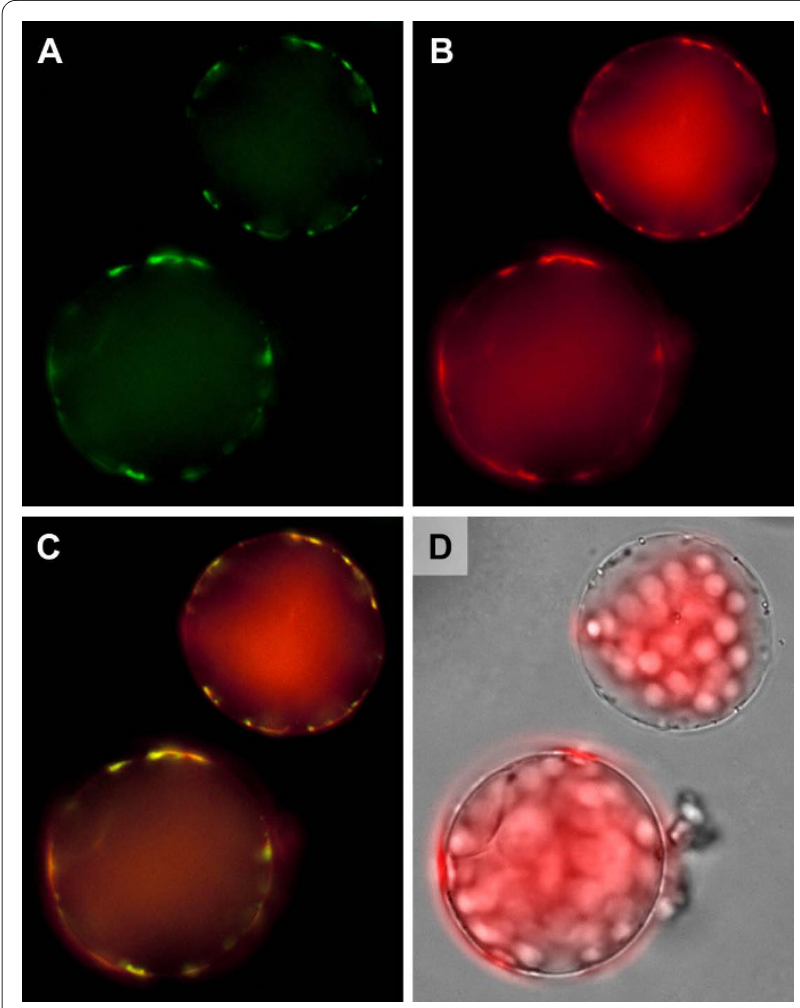

Figure 7 Subcellular localisation of ProDH2. False colour images of protoplasts isolated from Arabidopsis plants stably transformed with a 35S:ProDH2:GFP fusion construct and stained with MitoTracker Orange. A: GFP fluorescence depicted in green; B: MitoTracker fluorescence depicted in red; C: Overlay of A and B demonstrating co-localisation of ProDH2-GFP with mitochondria. D: Overlay of a transmitted light picture with chlorophyll autofluorescence.

sion levels are the main determinants of the different functionality of the two ProDH isoforms in Arabidopsis.

\section{Discussion}

Functional expression of Arabidopsis ProDHs in yeast

In the initial characterisation of ProDH1, two groups reported conflicting results concerning the expression of a functional protein from the native Arabidopsis gene in yeast. The contra group circumvented the problem by using the mTP of the yeast ProDH gene Put1, which might in itself interfere with the functionality test [25]. The data provided by the pro group lack proper positive and negative controls, for which it is impossible to tell if there is some strain specificity in the use of non-native mTPs [30]. Both studies suffer from the use of tryptophan auxotrophic strains, which necessitated the addition of low amounts of tryptophan, a potential nitrogen source. We circumvented these limitations by generating a $\Delta p u t 1$ deletion strain in the 23344c background, which is wildtype except for the ura3 mutation that served as a selec- 


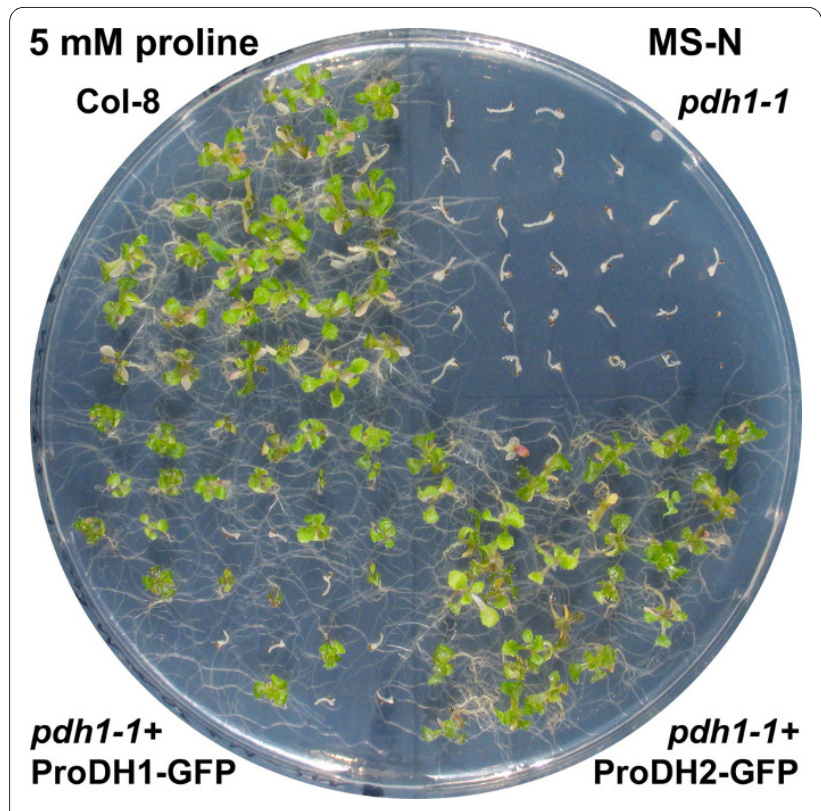

Figure 8 ProDH-GFP expression rescues the pdh1-1 mutant. Wildtype Col-8, pdh1-1 and pdh1-1 transformed with 35S:ProDH1:GFP or 35S:ProDH2:GFP were cultivated for 3 weeks on MS-N medium with 30 $\mathrm{mM}$ sucrose and $5 \mathrm{mM}$ Pro as the only source of nitrogen. The transformants are segregating $T_{2}$ populations, therefore not all seedlings are able to utilise Pro as nitrogen source.

tion marker for the expression plasmids. In this background, the native cDNAs of either ProDH gene from Arabidopsis did not confer sufficient ProDH activity to enable Pro utilisation. Exchange of the predicted mTPs for the N-terminus of a metabolically unrelated mitochondrial protein from yeast allowed the expression of functional ProDHs as evidenced by complementation of the Pro utilisation defect of the $\Delta p u t 1$ strain. From this we concluded that both ProDH genes from Arabidopsis encode proteins that mediate Pro catabolism to P5C/ GSA. Functional expression of the hybrid genes in yeast indicated that protein folding and co-factor insertion of the Arabidopsis proteins worked correctly in the heterologous system. Mitochondrial localisation of ProDH1GFP and ProDH2-GFP fusion proteins in Arabidopsis demonstrated functionality of the ProDH-mTPs in the native organism. Complementation of the Pro hypersensitive phenotype of Arabidopsis pdh1-1 mutant by overexpression of ProDH1-GFP or ProDH2-GFP provided further evidence that both proteins have the same enzymatic activity. It remains to be investigated, why the native Arabidopsis ProDH genes do not enable the expression of sufficient amounts of active and correctly targeted protein, whereas the Arabidopsis $P 5 C D H$ gene produced a functional protein with its native MTP [19].

\section{Spatial, temporal and metabolic regulation of ProDH2 expression}

Analysis of the tissue specificity of ProDH2 expression by promoter-Gus fusion demonstrated that ProDH2 expression is restricted to the vascular tissue. This finding is in agreement with regulation of $\mathrm{ProDH} 2$ expression by the transcription factor bZIP11, which is also spatially confined to the vasculature $[24,31]$. Translation of bZIP11 is repressed by sucrose, which resulted in reduced transcript levels of ProDH2 in the presence of high sucrose concentrations. High sucrose concentrations (3\%) might also be the reason for the absence of $\mathrm{ProDH} 2$ transcripts in the root-derived heterotrophic cell culture. The medium of the mixotrophic leaf-derived cell culture contained $1 \%$ sucrose (roughly corresponding to $30 \mathrm{mM}$ ), which is not sufficient to switch off ProDH2 expression. ProDH2-promoter driven Gus expression increased with leaf age and was strongest in the abscission zone of the floral organs, indicating that developmental signals also play a role in ProDH2 regulation. ProDH1, which had also enhanced promoter activity in the abscission zone, was additionally strongly expressed in pollen, in the stigma and in developing embryos, where no ProDH2 expression was detected [17]. In roots, both ProDH1 and ProDH2 were strongly detected, while $P 5 C D H$ was only weakly expressed. Potentially, arginine catabolism, which is the second task of P5CDH, is less prominent in roots. Alternatively, the strong ProDH expression could result from the preparation of the roots by extensive washing in (hypotonic) tap water. Expression changes of ProDH1 are reportedly very fast, while regulation of $P 5 C D H$ was slower [25,32]. Increased ProDH2 expression in old leaves was confirmed by northern blot analysis and evaluation of publicly available microarray data $[28,29]$. The spatial expression pattern was the most striking difference between ProDH1 and ProDH2 expression but also $\mathrm{NaCl}$ treatment caused down-regulation only of ProDH1. Similarly, Ribarits et al. (2007) found a differential regulation of two ProDH isoforms during dehydration in tobacco, while under these conditions the Arabidopsis ProDHs were co-regulated [23]. Over the broad range of different treatments documented in the microarray data collection, co-regulation of ProDH1 and ProDH2 is only sparsely observed, supporting our findings that the two ProDH isoforms fulfil at least partially different physiological functions. Up-regulation of $\mathrm{ProDH} 2$ expression during salt or drought stress might indicate a special need for proline degradation in the vasculature, while downregulation of ProDH1 occurs in the remaining tissues and enables proline accumulation. These findings imply that proline accumulation might not be favourable in the vascular tissue and the continued degradation of proline may 
provide energy and glutamate for other metabolic pathways or for long distance transport.

\section{ProDH2 cannot fully compensate the lack of ProDH1}

Loss-of-function mutants of ProDH1 were identified and characterised as sensitive to external Pro supply and heat treatment $[20,33]$. The existence and potential activity of $\mathrm{ProDH} 2$ was neglected in these studies. Mutants in $P 5 C D H$, which is a single copy gene in Arabidopsis, were completely unable to degrade Pro [16]. In a direct comparison of $p d h 1, p d h 2$ and $p 5 c d h$ mutants, we observed that $p d h 1$ and $p 5 c d h$ mutants were unable to utilise Pro as the sole nitrogen source, whereas $p d h 2$ mutants grew equally well as the corresponding wildtype. When $p d h 1-$ 1 , $p d h 1-4$ and $p 5 c d h-2$ mutants were grown on medium containing mineral nitrogen, only $p 5 c d h-2$ was more sensitive to growth-inhibition by external Pro than the wildtype. Preliminary characterisation of the pdh2-1 mutant showed a slightly enhanced Pro accumulation in response to salt stress but not after external Pro application (data not shown). Pro sensitivity and stress induced Pro accumulation differed between the Col-8 and Ler wildtypes, confirming that a useful double mutant can only be produced after extensive backcrossing. From these data we conclude that the slightly enhanced ProDH2 expression in Pro treated $p d h 1$ mutants could not fully compensate the loss of ProDH1, while it may be able to diminish the toxic effects of Pro. Alternatively, the toxic effects of Pro supply might result from $\mathrm{P} 5 \mathrm{C}$, which is only expected to accumulate in the $p 5 c d h-2$ mutant. Recent results presented by Miller et al. (2009) suggest that ROS production by ProDH rather than P5C accumulation is the cause of the toxic effects of Pro application [22]. Unfortunately, Miller et al. (2009) did not analyse ProDH mutants and from the data presented it is not possible to determine, whether ROS production is the cause or a consequence of the loss of mitochondrial integrity that was observed in earlier studies [21]. Our identification of $p d h 2$ mutants and the possibility to generate double mutants completely devoid of ProDH activity opens new possibilities to dissect the contribution of Pro degradation to Pro toxicity and stress tolerance. The detailed characterisation of the pdh2 mutants will also help to identify the specific physiological function of ProDH2 in the vascular tissue.

\section{Conclusions}

Despite numerous biochemical and molecular approaches, the protective mechanism of Pro accumulation remains unclear. Each new piece of experimental evidence added to our picture of Pro metabolism in plants requires careful re-interpretation of previous results. Only by a thorough analysis of all components and also non-obvious side effects it will become possible to understand the seemingly dual nature of Pro as a protective and toxic compound. The functional characterisation of ProDH2 opens up a whole new set of experimental approaches to understand the function of Pro in the stress tolerance of plants.

\section{Methods}

\section{Plant material and growth conditions}

Arabidopsis (Arabidopsis thaliana (L.) Heynh. ecotype Col-8 or Ler) and T-DNA or transposon insertion lines were obtained from the NASC (SALK_108179, GABI_918D08, SAIL_90_G05, GABI_328G05 (pdh2-2), GT1788 (pdh2-1), SALK_081276 (pdh1-2), GABI_308F08 (pdh1-3), SALK_119334 (pdh1-4) and Salk_018453 $(p 5 c d h-2))$, from Bernd Weisshaar (GABI_187C05; GABI-Kat program, Cologne, Germany [34], or from the KAZUSA research institute, Kisarazu, Japan (pdh1-1). Presence of the T-DNA and allelic status were verified by PCR and sequencing of the T-DNA flanking sequences. Gene and T-DNA-specific primers are listed in Table 1. All physiological experiments with $p d h 1$ and $p 5 c d h$ mutants were performed with homozygous progeny of plants backcrossed three times to $\mathrm{Col}-8$. For the $p d h 2-1$ mutant (in Ler background), segregation analysis demonstrated the presence of a single transposon. Plants were cultivated axenically in $9 \mathrm{~cm}$ Petri dishes on commercial MS medium (Duchefa, Haarlem, Netherlands) or selfmade MS medium, in which $\mathrm{KNO}_{3}$ and $\mathrm{NH}_{4} \mathrm{NO}_{3}$ were replaced by $20 \mathrm{mM} \mathrm{KCl} \mathrm{[35].} \mathrm{Media} \mathrm{were} \mathrm{supplemented}$ with sucrose and nitrogen containing compounds as indicated for each experiment and solidified with $8 \mathrm{~g} / \mathrm{l}$ purified agar (BD Biosciences, San Jose, CA, USA) when appropriate. Seeds were surface sterilised by sequential treatment with $70 \%(\mathrm{v} / \mathrm{v}) \mathrm{EtOH}$ and $1 \%(\mathrm{w} / \mathrm{v}) \mathrm{NaOCl} /$ $0,01 \%(\mathrm{v} / \mathrm{v})$ Triton-X-100 and vernalised for $24 \mathrm{~h}$ at $4^{\circ} \mathrm{C}$ in $0.1 \%(\mathrm{w} / \mathrm{v})$ agarose. Plants were cultivated in a climatised room with short day $(9 \mathrm{~h})$ light period and a light intensity of $110 \mu \mathrm{mol}$ photons ${ }^{*} \mathrm{~s}^{-1}{ }^{*} \mathrm{~m}^{-2}$ from mixed fluorescence tubes (Biolux and Fluora, Osram, Munich, Germany) at a constant temperature of $23^{\circ} \mathrm{C}$. For induction experiments, axenically grown seedlings were transferred to liquid medium and incubated in the light with agitation at $100 \mathrm{rpm}$ for $6 \mathrm{~h}$. Induction in liquid medium was chosen because wildtype plants metabolised proline very quickly when it was provided via the solid culture medium and only marginal increases in proline content were observed in the leaves. For seed production, plants were kept in a greenhouse with a light period of at least $16 \mathrm{~h}$. A heterotrophic (white) culture was obtained from Giuseppe Forlani, Ferrara, Italy and maintained in liquid MS medium supplemented with $3 \%(\mathrm{w} / \mathrm{v})$ sucrose, $1 \times$ Gamborg's vitamin mix, $0.5 \mathrm{mg} / \mathrm{l}$ 2,4-dichlorophenoxyacetic acid, $0.5 \mathrm{mg} / \mathrm{l}$ benzylaminopurine and $0,2 \%(\mathrm{v} / \mathrm{v})$ Plant Preservative Medium (Plant Cell Technology, Washington, DC, USA) under constant agitation in dim 
Table 1: PCR primers used in this study

\begin{tabular}{|c|c|c|}
\hline Name1 & Sequence (5'->3') & Used for \\
\hline Pdh1-LP1 (A) & tctcctctatcccaacctctg & $\begin{array}{l}\text { pdh1-1, GABI_308F08 (pdh1-3), } \\
\text { SALK_119334 (pdh1-4) }\end{array}$ \\
\hline Pdh1-RP1 (B) & gatcgctcactcgtttcagaag & \\
\hline Pdh1-LP2 (C) & aagttggtgagaggggcttac & SALK_081276 (pdh1-2) \\
\hline Pdh1-NS-r (D) & cgcaatcccggcgattaatctc & ProDH1 cloning \\
\hline Pdh1-f & caccataATGgcaacccgtcttctc ${ }^{2}$ & \\
\hline Pdh2-LP & gtaaccagcccctaaacctc & $\begin{array}{l}\text { SALK_108179, GABI_918D08, } \\
\text { SAIL_90_G05 }\end{array}$ \\
\hline Pdh2-RP & ggagtactagatcgcgtgtaac & \\
\hline Pdh2-LP2 (F) & aaaccctaccttcgtctcac & GT1788 (pdh2-1), GABI_328G05 (pdh2-2) \\
\hline Pdh2-RP2 (G) & cactaacccgttttaggacattc & \\
\hline Pdh2-LP3 & gagaagagttatggcttggtg & GABI_187C05 \\
\hline Pdh2-RP3 & atgtccctttgtaatctgaattgg & \\
\hline Pdh2-f (E) & caccataATGgcaaaccgtttcctc ${ }^{2}$ & ProDH2 cloning \\
\hline Pdh2-NSr & ccaagccataactcttctcttaag & \\
\hline Pdh2-Prom-f & catttggatccttaccatccac & ProDH2 promoter cloning \\
\hline Pdh2-Prom-r & cgggatccgtttgcCATttaaactc ${ }^{2}$ & \\
\hline Salk_LBb2 & ttcggaaccaccatcaaacag & SALK lines \\
\hline Gabi-LB & cccatttggacgtgaatgtagacac & GABI lines \\
\hline Sail-LB2 & gcttcctattatatcttcccaaattaccaataca & SAIL_90_G05 \\
\hline Gus5'rev & atttcacgggttggggtttc & GT1788 \\
\hline Put1KO-f & $\begin{array}{l}\text { aaacatcgctacatagtaataacactaacgcacgcta } \\
\text { gaaCGGATCCCCGGGTTAATTAA }\end{array}$ & Put1 knockout \\
\hline Put1KO-r & $\begin{array}{l}\text { ttggtttgtctttgaaattggagtatatattatagtcctcG } \\
\text { AATTCGAGCTCGTITAAAC } 3\end{array}$ & \\
\hline $\mathrm{SDH}-\mathrm{f}$ & caccacgagaataaagATGctatcgct ${ }^{2}$ & SDH-MTP \\
\hline SDH-Pdh1-r & $\begin{array}{l}\text { cttgttgtccaaaggagagCTCGTGATCTATTAT } \\
\text { GTG }^{4}\end{array}$ & SDH-MTP ProDH1 fusion \\
\hline SDH-Pdh2-r & $\begin{array}{l}\text { ggttggtcaaaggaaaggatCTCGTGATCTATTA } \\
\text { TGTG }^{4}\end{array}$ & SDH-MTP ProDH2-fusion \\
\hline Put1-f & caccctagaaATGatagcttcc $^{2}$ & Put1 cloning \\
\hline Put1-r & taggcctactctttttggaatc & \\
\hline Pdh1-pr-r & atggtcataaaacgtacttttcac & $\begin{array}{l}\text { northern probes } \\
\text { (with Pdh1-f or Pdh2-f) }\end{array}$ \\
\hline
\end{tabular}


Table 1: PCR primers used in this study (Continued)

\begin{tabular}{|c|c|c|}
\hline Pdh2-pr-r & gactcatacacgctactcac & \\
\hline P5CS1-f & aatgagaggaaaaggacaag & $\begin{array}{l}\text { P5CS1 probe } \\
{[8]}\end{array}$ \\
\hline P5CS1-r & gataggatatgagtactaagcagag & \\
\hline P5CDH-f & tggacagaagtgttctgcac & $\mathrm{P} 5 \mathrm{CDH}$ probe \\
\hline $\mathrm{P} 5 \mathrm{CDH}-\mathrm{r}$ & gcttccaacactagaggaag & \\
\hline
\end{tabular}

light. A mixotrophic, green cell culture was obtained from Silke Robatzek (Cologne, Germany and maintained in liquid MS medium supplemented with $1 \%(\mathrm{w} / \mathrm{v})$ sucrose, $4 \times$ Gamborg's vitamin mix, 0,5 mg/l 2-(1-naphthyl)acetic acid and $0,1 \mathrm{mg} / \mathrm{l}$ kinetin at $110 \mu \mathrm{mol}$ photons $\mathrm{s}^{*} \mathrm{~s}^{-1} \mathrm{~m}^{-2}$.

\section{Promoter-GUS construct and histochemical analysis}

A 1500 bp promoter fragment including the native start codon of ProDH2 was amplified from Arabidopsis genomic DNA with primers introducing BamHI restriction sites (Tab. 1). The PCR-product was subcloned into pCR-blunt and sequenced. The promoter fragment was excised with BamHI and inserted into pCB308 [36]. Agrobacterium tumefaciens strain GV3101 was used for floral dip transformation of Arabidopsis [37,38]. Transformants were selected by spraying soil grown seedlings with $50 \mathrm{mg} / \mathrm{l} \mathrm{BASTA}$ or by addition of $10 \mathrm{mg} / \mathrm{l} \mathrm{BASTA}$ to the culture medium. Histochemical GUS staining was performed according to [39].

\section{Expression analysis}

Total RNA was extracted from snap-frozen material with phenol/guanidine thiocyanate reagent according to each manufacturer's recommendations. Per lane, $15 \mu \mathrm{g}$ of total RNA was separated by denaturing agarose gel electrophoresis and transferred to a positively charged nylon membrane by capillary transfer. ProDH1, ProDH2, P5CS1 and $P 5 C D H$ transcripts were detected by hybridisation with digoxigenin-labelled PCR products obtained with primers listed in Tab. 1, followed by detection with alkaline phosphatase coupled anti-DIG antibodies and the chemiluminescent substrate CDP-star (Roche, Basel, Switzerland). Densitometric quantification was performed with the ImageJ software. For RT-PCR, $4 \mu \mathrm{g}$ of total RNA was converted to cDNA using random hexamer primers and the Transcriptor cDNA synthesis Kit (Roche). One $\mu \mathrm{l}$ of the cDNA preparation was used as a template for endpoint RT-PCR. Public microarray data collection were evaluated with the Expression Browser of the Bio-Array Resource and Genvestigator v3 $[28,29,40,41]$.

\section{ProDH1-GFP and ProDH2-GFP constructs and imaging}

The open reading frames of ProDH1 and ProDH2 without the stop codon were amplified by PCR from EST clones 38H5 and full-length ORF clone U66465, respectively (ABRC, Columbus, OH, USA). Sequences of PCR primers are given in Tab. 1 . The resulting PCR fragments were purified and integrated into pENTR by directional TOPO cloning (Invitrogen, Carlsbad, CA, USA). Subsequently, the ProDH1 and ProDH2 cDNAs were transferred from pENTR to pEarleyGate103 (CD3-685, ABRC) or pGWB5 [42] by LR-recombination (Invitrogen). Both plant transformation vectors yielded the same results. Sequencing of the resulting constructs demonstrated inframe fusion of the ProDH cDNAs to the GFP gene and revealed that EST $38 \mathrm{H} 5$ is not derived from Col-0 but most likely from C24 ecotype, which carries 12 silent or conservative single nucleotide polymorphisms in the ProDH1 coding sequence. These nucleotide exchanges are present in a number of Arabidopsis wildtype accessions. Transgenic plants were produced as described above except for selection of plants that carried pGWB5 derived constructs, which was performed in axenic culture.

Protoplasts from transformed leaves were obtained by overnight incubation with cellulase and macerase (Serva, Heidelberg, Germany), stained with MitoTracker Orange (Invitrogen) and viewed under an Olympus BX51 epifluorescence microscope equipped with a Nikon DXM1200 digital camera system (Olympus Europe, Hamburg, Germany). Chlorophyll autofluorescence, MitoTracker and GFP fluorescence of the cells were dissected using the filter sets U-MWSG2 (Olympus), 41007 and 41020 (Chroma Technology Corp, Rockingham, VT, USA), respectively. False colouring and overlay of images was performed using AxioVision software (Zeiss, Oberkochen, Germany). 
Yeast strains, growth conditions and expression plasmids Saccharomyces cerevisiae strain 23344c (Mata ura3; [43]) was received from Giuseppe Forlani, Ferrara, Italy. For the deletion of the Put1 gene, the kanamycin resistance cassette from pFA6a-KanMX6 was amplified with primers adding 40 bp of Put1 $5^{\prime}$ and 3 ' sequences (Tab. 1, [44]). After transformation of $23344 \mathrm{c}$ with the PCR product, geneticin resistant colonies were selected and tested for their ability to utilise Pro or urea as the sole source of nitrogen. Replacement of the Put1 coding sequence by the resistance cassette was verified by PCR. The Put 1 coding sequence and the Sdh1-mTP (bases 1-156 of the coding sequence) were amplified with the primers listed in Tab. 1 from genomic DNA isolated from 23344c. The reverse primers for the $S d h 1$-mTP introduce overlaps to the coding sequences of ProDH1 and ProDH2 that were used to fuse the coding sequences by PCR. All final PCR products were introduced into pENTR as described above and the coding sequences were transferred to pYES-Dest52 by LR-recombination (Invitrogen). Plasmids were introduced into yeast by the LiAc/PEG method [45] and transformants were selected on synthetic medium with $20 \mathrm{~g} / \mathrm{l}$ glucose and $5 \mathrm{~g} / \mathrm{l} \mathrm{NH}_{4} \mathrm{SO}_{4}$ supplemented with a mixture of amino acids but no uracil (BD biosciences). For growth tests on organic nitrogen sources, yeast strains were washed once in sterile water, resuspended in $0.1 \%$ agarose and streaked on plates containing synthetic minimal medium, $20 \mathrm{~g} / \mathrm{l}$ galactose and no other additives except the indicated nitrogen sources.

\section{Additional material}

Additional file 1 ProDH1-GFP is localised in mitochondria. False colour images of a protoplast expressing a ProDH1-GFP fusion protein under control of the CaMV-35S promoter and stained with MitoTracker Orange.

\section{Authors' contributions}

GM analysed the expression pattern of ProDH2. SE generated the yeast expression constructs. DF designed the study, performed the other experiments and compiled the manuscript. All authors contributed to and approved the final manuscript.

\section{Acknowledgements}

We would like to thank Giuseppe Forlani, University of Ferrara, Italy, for communicating unpublished results, for the $23344 \mathrm{c}$ yeast strain, for the white Arabidopsis cell culture and for helpful comments on the manuscript. Marc Erhardt, University of Konstanz, is acknowledged for the pFA6a-KanMX6 plasmid and for helpful advice on the construction of the $\Delta$ put 7 knockout strain. We are also thankful to Tsuyoshi Nakagawa, Shimane University, Japan, for the pGWB5 vector. Regina Grimm, Silvia Kuhn, Roswitha Miller-Sulger and the gardeners of the University of Konstanz are acknowledged for technical assistance in the lab and for excellent plant care, respectively. We are indebted to Iwona Adamska for providing a functional working environment and for critical reading of the manuscript. Additional thanks to Helen Gunter, who made helpful suggestions concerning the use of the English language. The University of Konstanz provided financial support for this study and GM is grateful for a fellowship by the Stiftung der deutschen Wirtschaft.

\section{Author Details}

Department of Plant Physiology and Biochemistry, Biology Section, University of Konstanz, Universitätsstrasse 10, 78464 Konstanz, Germany

Received: 16 November 2009 Accepted: 19 April 2010 Published: 19 April 2010

\section{References}

1. Mani S, Cotte B Van De, Van Montagu M, Verbruggen N: Altered levels of proline dehydrogenase cause hypersensitivity to proline and its analogs in Arabidopsis. Plant Physiol 2002, 128(1):73-83.

2. Nanjo T, Kobayashi M, Yoshiba Y, Sanada Y, Wada K, Tsukaya H, Kakubari Y, Yamaguchi-Shinozaki K, Shinozaki K: Biological functions of proline in morphogenesis and osmotolerance revealed in antisense transgenic Arabidopsis thaliana. Plant J 1999, 18(2):185-193.

3. Szekely G, Abraham E, Cseplo A, Rigo G, Zsigmond L, Csiszar J, Ayaydin F, Strizhov N, Jasik J, Schmelzer E, et al:: Duplicated P5CS genes of Arabidopsis play distinct roles in stress regulation and developmental control of proline biosynthesis. Plant Journal 2008, 53(1):11-28.

4. Verbruggen $N$, Hermans C: Proline accumulation in plants: a review. Amino Acids 2008, 35(4):753-759.

5. Szabados L, Savoure A: Proline: a multifunctional amino acid. Trends Plant Sci 2010, 15(2):89-97.

6. Hare PD, Cress WA: Metabolic implications of stress-induced proline accumulation in plants. Plant Growth Regulation 1997, 21(2):79-102.

7. Phang JM, Donald SP, Pandhare J, Liu Y: The metabolism of proline, a stress substrate, modulates carcinogenic pathways. Amino Acids 2008, 35(4):681-690.

8. Strizhov N, Abraham E, Okresz L, Blickling S, Zilberstein A, Schell J, Koncz C, Szabados L: Differential expression of two P5CS genes controlling proline accumulation during salt-stress requires $A B A$ and is regulated by ABA1, ABI1 and AXR2 in Arabidopsis. Plant J 1997, 12(3):557-569.

9. Mattioli R, Falasca G, Sabatini S, Altamura MM, Costantino P, Trovato M: The proline biosynthetic genes P5CS1 and P5CS2 play overlapping roles in Arabidopsis flower transition but not in embryo development. Physiologia Plantarum 2009, 137(1):72-85

10. Rayapati PJ, Stewart CR, Hack E: Pyrroline-5-Carboxylate Reductase Is in Pea (Pisum sativum L.) Leaf Chloroplasts. Plant Physiol 1989, 91(2):581-586.

11. Szoke A, Miao GH, Hong Z, Verma DP: Subcellular Location of DeltaPyrroline-5-Carboxylate Reductase in Root/Nodule and Leaf of Soybean. Plant Physiol 1992, 99(4):1642-1649.

12. Adams E, Frank L: Metabolism of proline and the hydroxyprolines. Annu Rev Biochem 1980, 49:1005-1061.

13. Funck D, Stadelhofer B, Koch W: Ornithine-delta-aminotransferase is essential for arginine catabolism but not for proline biosynthesis. $B M C$ Plant Biol 2008, 8:40

14. Elthon TE, Stewart CR: Proline Oxidation in Corn Mitochondria: Involvement of NAD, Relationship to Ornithine Metabolism, and Sidedness on the Inner Membrane. Plant Physiol 1982, 70(2):567-572.

15. Elthon TE, Stewart CR: Submitochondrial Location and Electron Transport Characteristics of Enzymes Involved in Proline Oxidation. Plant Physiol 1981, 67(4):780-784

16. Deuschle K, Funck D, Forlani G, Stransky H, Biehl A, Leister D, Graaff E van der, Kunze R, Frommer WB: The role of [Delta]1-pyrroline-5-carboxylate dehydrogenase in proline degradation. Plant Cell 2004, 16(12):3413-3425

17. Nakashima K, Satoh R, Kiyosue T, Yamaguchi-Shinozaki K, Shinozaki K: A gene encoding proline dehydrogenase is not only induced by proline and hypoosmolarity, but is also developmentally regulated in the reproductive organs of Arabidopsis. Plant Physiol 1998, 118(4):1233-1241

18. Borsani O, Zhu J, Verslues PE, Sunkar R, Zhu JK: Endogenous siRNAs derived from a pair of natural cis-antisense transcripts regulate salt tolerance in Arabidopsis. Cell 2005, 123(7):1279-1291.

19. Deuschle K, Funck D, Hellmann H, Daschner K, Binder S, Frommer WB: A nuclear gene encoding mitochondrial Delta-pyrroline-5-carboxylate dehydrogenase and its potential role in protection from proline toxicity. Plant J 2001, 27(4):345-356 
20. Nanjo T, Fujita M, Seki M, Kato T, Tabata S, Shinozaki K: Toxicity of free proline revealed in an arabidopsis T-DNA-tagged mutant deficient in proline dehydrogenase. Plant Cell Physiol 2003, 44(5):541-548.

21. Hare PD, Cress WA, van Staden J: Disruptive effects of exogenous proline on chloroplast and mitochondrial ultrastructure in Arabidopsis leaves. South African Journal of Botany 2002, 68(3):393-396.

22. Miller G, Honig A, Stein H, Suzuki N, Mittler R, Zilberstein A: Unraveling delta1-pyrroline-5-carboxylate-proline cycle in plants by uncoupled expression of proline oxidation enzymes. J Biol Chem 2009, 284(39):26482-26492.

23. Ribarits A, Abdullaev A, Tashpulatov A, Richter A, Heberle-Bors E, Touraev $A$ : Two tobacco proline dehydrogenases are differentially regulated and play a role in early plant development. Planta 2007, 225(5):1313-1324

24. Hanson J, Hanssen M, Wiese A, Hendriks MM, Smeekens S: The sucrose regulated transcription factor bZIP11 affects amino acid metabolism by regulating the expression of ASPARAGINE SYNTHETASE1 and PROLINE DEHYDROGENASE2. Plant J 2008, 53(6):935-949.

25. Kiyosue T, Yoshiba Y, Yamaguchi-Shinozaki K, Shinozaki K: A nuclear gene encoding mitochondrial proline dehydrogenase, an enzyme involved in proline metabolism, is upregulated by proline but downregulated by dehydration in Arabidopsis. Plant Cell 1996, 8(8):1323-1335.

26. Athena promoter visualization tool [http:// www.bioinformatics2.wsu.edu/Athena

27. O'Connor TR, Dyreson C, Wyrick JJ: Athena: a resource for rapid visualization and systematic analysis of Arabidopsis promoter sequences. Bioinformatics 2005, 21(24):4411-4413.

28. BAR The Bio-Array Resource for Arabidopsis Functional Genomics [http://bbc.botany.utoronto.ca/

29. Genevestigator v3 [http://www.genevestigator.com]

30. Peng Z, Lu Q, Verma DP: Reciprocal regulation of delta 1-pyrroline-5carboxylate synthetase and proline dehydrogenase genes controls proline levels during and after osmotic stress in plants. Mol Gen Genet 1996, 253(3):334-341

31. Rook F, Gerrits N, Kortstee A, van Kampen M, Borrias M, Weisbeek $P$, Smeekens S: Sucrose-specific signalling represses translation of the Arabidopsis ATB2 bZIP transcription factor gene. Plant J 1998, 15(2):253-263.

32. Hellmann $H$, Funck D, Rentsch D, Frommer WB: Hypersensitivity of an Arabidopsis sugar signaling mutant toward exogenous proline application. Plant Physiol 2000, 123(2):779-789.

33. Larkindale J, Vierling E: Core genome responses involved in acclimation to high temperature. Plant Physio/ 2008, 146(2):748-761.

34. Rosso MG, Li Y, Strizhov N, Reiss B, Dekker K, Weisshaar B: An Arabidopsis thaliana T-DNA mutagenized population (GABI-Kat) for flanking sequence tag-based reverse genetics. Plant Mol Biol 2003, 53(12):247-259.

35. Murashige T, Skoog F: A revised medium for rapid growth and bioassays with tobacco tissue cultures. Physiol Plant 1962, 15(15):473-497.

36. Xiang C, Han P, Lutziger I, Wang K, Oliver DJ: A mini binary vector series for plant transformation. Plant Mol Biol 1999, 40(4):711-717.

37. Clough SJ, Bent AF: Floral dip: a simplified method for Agrobacteriummediated transformation of Arabidopsis thaliana. Plant J 1998, 16(6):735-743

38. Wydro M, Kozubek E, Lehmann P: Optimization of transient Agrobacterium-mediated gene expression system in leaves of Nicotiana benthamiana. Acta Biochim Pol 2006, 53(2):289-298.

39. Martin T, Hellmann H, Schmidt R, Willmitzer L, Frommer WB: Identification of mutants in metabolically regulated gene expression. The Plant Journal 1997, 11(1):53-62.

40. Hruz T, Laule O, Szabo G, Wessendorp F, Bleuler S, Oertle L, Widmayer P, Gruissem W, Zimmermann P: Genevestigator v3: a reference expression database for the meta-analysis of transcriptomes. Adv Bioinformatics 2008, 2008:420747.

41. Toufighi K, Brady SM, Austin R, Ly E, Provart NJ: The Bio-Array Resource for Plant Functional Genomics. Plant J 2005, 43(1):153-163.

42. Nakagawa T, Kurose T, Hino T, Tanaka K, Kawamukai M, Niwa Y, Toyooka K, Matsuoka K, Jinbo T, Kimura T: Development of series of gateway binary vectors, pGWBs, for realizing efficient construction of fusion genes for plant transformation. Journal of Bioscience and Bioengineering 2007, 104(1):34
43. Grenson M: The utilization of exogenous pyrimidines and the recycling of uridine-5'-phosphate derivatives in Saccharomyces cerevisiae, as studied by means of mutants affected in pyrimidine uptake and metabolism. Eur J Biochem 1969, 11(2):249-260.

44. Achim W, Arndt B, Rainer P, Peter P: New heterologous modules for classical or PCR-based gene disruptions in Saccharomyces cerevisiae. Yeast 1994, 10(13):1793-1808.

45. Gietz RD, Woods RA: Transformation of yeast by lithium acetate/singlestranded carrier DNA/polyethylene glycol method. Methods Enzymol 2002, 350:87-96

doi: 10.1186/1471-2229-10-70

Cite this article as: Funck et al., Non-redundant functions of two proline dehydrogenase isoforms in Arabidopsis BMC Plant Biology 2010, 10:70

\section{Submit your next manuscript to BioMed Centra and take full advantage of:}

- Convenient online submission

- Thorough peer review

- No space constraints or color figure charges

- Immediate publication on acceptance

- Inclusion in PubMed, CAS, Scopus and Google Scholar

- Research which is freely available for redistribution 